\title{
Acute liver failure precipitated by acute Budd-Chiari syndrome and complete portal vein thrombosis
}

\author{
Yuji Suzuki $^{1}$, Akiko Suzuki ${ }^{2}$, Keisuke Kakisaka ${ }^{3}$, and Yasuhiro Takikawa ${ }^{4}$ \\ ${ }^{1}$ iwate medical university \\ ${ }^{2}$ Iwate Medical University \\ ${ }^{3}$ Iwate Medical University School of Medicine \\ ${ }^{4}$ Iwate Medical University
}

February 3, 2021

\section{Acute liver failure precipitated by acute Budd-Chiari syndrome and complete portal vein thrombosis}

Yuji Suzuki MD, PhD; Akiko Suzuki MD, PhD; Keisuke Kakisaka MD, PhD; Yasuhiro Takikawa MD, PhD

Division of Hepatology, Department of Internal Medicine, Iwate Medical University School of Medicine, Yahaba-cho, Iwate, Japan

\section{Address for correspondence:}

Yuji Suzuki, MD, PhD

Division of Hepatology, Department of Internal Medicine, Iwate Medical University School of Medicine

1-1-1 Idaidori, Yahaba-cho, Shiwa-gun, Iwate 028-3694, Japan

Tel: +81-19-651-5111; Fax: +81-19-907-7166

E-mail:yusuzuki@iwate-med.ac.jp

Funding: The authors received no financial support for the research, authorship, and/or publication of this article.

Conflict of Interest: The authors declared no potential conflict of interest with respect to the research, authorship, and/or publication of this article.

Patient Consent: Informed consent was obtained from the patient to publish his case and medical images.

The total number of figures: 2

Acknowledgments: None

Word count : 238

A 46-year-old man complaining of fatigue and right upper quadrant pain that lasted for 10 days was referred to our hospital. Physical examination revealed abdominal distention. Contrast-enhanced computed tomography revealed complete thrombotic occlusion of the main trunk of the portal and splenic veins (Figure $1 \mathrm{~A}$, arrows), as well as the three main hepatic veins (Figure 1B, arrows). Laboratory findings revealed leukocytosis (leukocytes, $25,610 / \mu \mathrm{L}$ ) and acute liver failure (alanine aminotransferase, 3,188 IU/L; aspartate transaminase, 3,804 IU/L; total bilirubin, $2.4 \mathrm{mg} / \mathrm{dL}$; ammonia, $140 \mu \mathrm{g} / \mathrm{dL}$; and international normalized ratio, 2.24). Although low-molecular-weight heparin was administered subcutaneously, the patient developed 
grade III hepatic encephalopathy 5 days after admission. Acute liver failure precipitated by acute BuddChiari syndrome and complete portal vein thrombosis was diagnosed. ${ }^{1}$ Ten days after admission, emergency orthotopic liver transplantation was performed. Microscopic examination of the explanted liver showed massive hepatocyte loss in the centrilobular region (Figure 2, hematoxylin and eosin staining, 10×). The patient was eventually diagnosed with JAK2 V617F mutation-positive myeloproliferative neoplasm and was treated with hydroxyurea and aspirin, following liver transplantation. ${ }^{2}$ Over the 5 -year follow-up period, he remains well without post-transplant complications.

Myeloproliferative neoplasms, including JAK2 V617F mutations, are the most common cause of Budd-Chiari syndrome and non-malignant, non-cirrhotic portal vein thrombosis. ${ }^{3}$ We demonstrated that acute BuddChiari syndrome and complete portal vein thrombosis might occur simultaneously by myeloproliferative neoplasm, resulting in acute liver failure. Liver transplantation could be effective even in such a catastrophic condition.

\section{Figure Legends}

Figure 1. Coronal contrast-enhanced computed tomography in the portal venous phase shows decreased attenuation in the portal vein and splenic vein without any signs of cavernous transformation. Yellow arrows indicate thrombosis (A). Axial contrast-enhanced computed tomography in the portal venous phase shows decreased attenuating thrombus in the three main hepatic veins (arrows), compression of inferior vena cava, enlargement of the liver, and presence of ascites (B).

Figure 2. Explanted liver specimen shows massive hepatocyte loss in the centrilobular region (hematoxylin and eosin staining, original magnification $\times 10$ ).

\section{References}

1. Parekh J, Matei VM, Canas-Coto A, Friedman D, Lee WM; Acute Liver Failure Study Group. Budd-Chiari syndrome causing acute liver failure: a multicenter case series. Liver Transpl 2017;23:135-142.

2. Barbui T, Tefferi A, Vannucchi AM, et al. Philadelphia chromosome-negative classical myeloproliferative neoplasms: revised management recommendations from European LeukemiaNet. Leukemia 2018;32:10571069 .

3. Smalberg JH, Arends LR, Valla DC, Kiladjian JJ, Janssen HL, Leebeek FW. Myeloproliferative neoplasms in Budd-Chiari syndrome and portal vein thrombosis: a meta-analysis. Blood 2012;120:4921-4928.
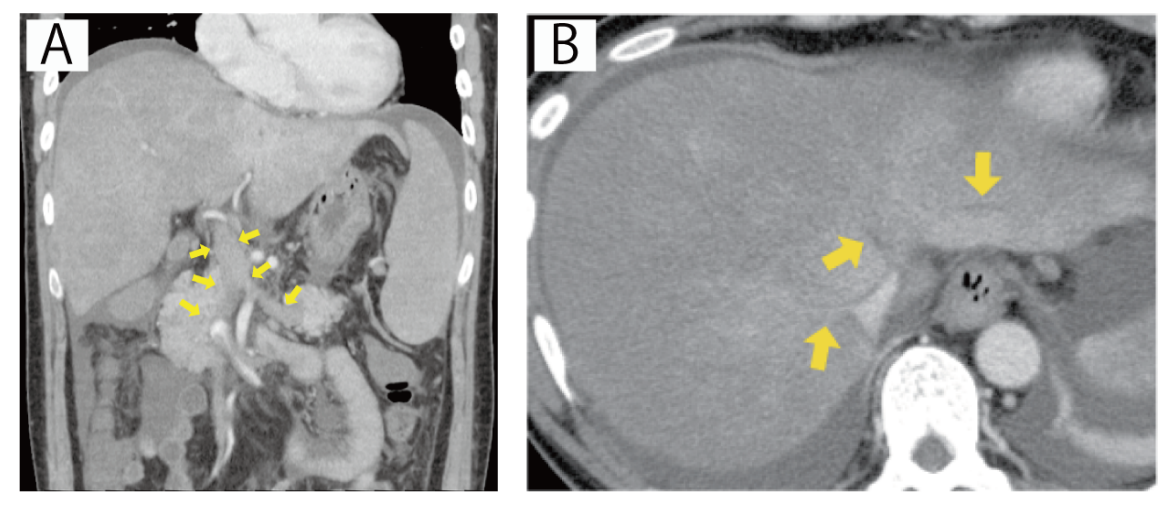


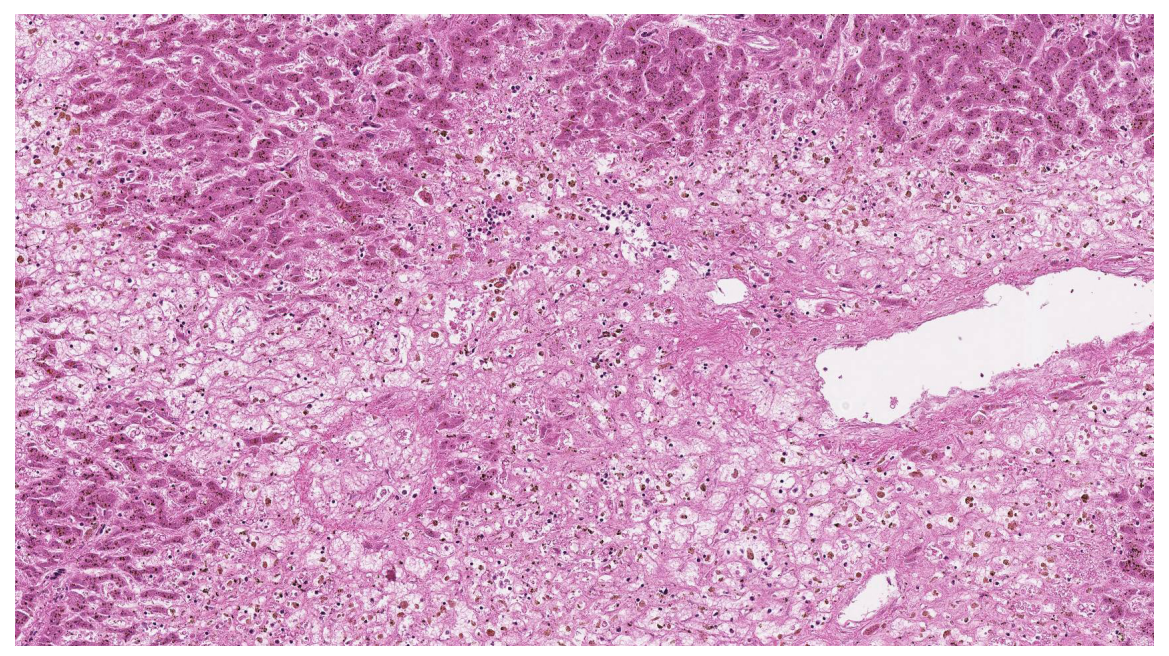

